\title{
Clasificación ¿Actividad Técnica o Teórica?
}

\author{
Susana Sander \\ Investigadora del CUIB
}

\section{RESUMEN}

LaClasificaciónBibliotecológicaes una ac ti vi dad teórica ma nifies ta en los distin tos sis te mas decla sificación ló gi cos- de duc tivos, lógicos-simbólicos y ló gi cos-mate máticos; asícomoen las relaciones abs trac tas que se ge ne ran en tre su je to y ob je to al po ner se en prác tica cualquier sistema de clasificación.

\section{ABSTRACT}

Library classification is a theoretical activity expressed in different classification systems: deductive-logical, symbolic- logical and mathe matical-logical; li kewi se in the abs tractrelations ge ne rated between the subject and the ob ject when any clas si fi ca tion sys tem is put in practice.

\section{Introducción}

La clasificación cons ti tu ye uno de los me dios fun damen tales para que la bi blio te ca al can ce sus obje ti vos so cia les. Por ello, in ten tar pen sar lo que es en sí mis ma la cla si fi ca ción bi blio tecológica, nos parece un ejercicio intelectual que pudiera repercutir positivamente en dicha capacidad mediadora.

De los varios caminos de reflexión sobre la clasificación bibliotecológica, el que consideramos más fértil, por ahora, es el que intenta plantear y dilucidar el problema del carácter técnico o teórico de esta actividad.

\section{EL PROBLEMA}

La cuestión del carácter técnico o teórico de la clasificación, no es gratuita. Una cierta profundización en su ámbito nos lleva, por un lado, a reconocer en ella una actividad práctica con ob je ti vos con cre tos muy cla ros y con proce di mien tos, reglas y normas que permitencalificarla, demanerainmediata, como una técnica. Sin embargo, en pocas ocasiones nos preguntamos ¿qué es técnica? ¿en qué radica lo técnico de esta actividad? Por otro lado, el enfoque sobre el contenido de la clasificación, como requisito primario para la realización de esa actividad, obliga a observar en ella un nivel autónomo cons ti tui do por es truc tu ras, sím bolos y ele men tos cog nos citivos y, por lo mis mo, esen cial men te abs trac tos que, sin lu gar a dudas, permiten designarla como una actividad teórica. Empero, cuándo calificamos como técnica a la clasificación, y cuándo la calificamos como teoría, nos referimos a una misma actividad en distintas dimensiones.

\section{DE LO TECNICO}

Si la cla si fi ca ción es una téc ni ca, ne ce si ta mos pre ci sar quées técnica. Por principio, toda técnica es una práctica pero no toda prác ti ca es téc ni ca. Exis te la prác ti ca teórica. Prác ti ca es praxis: produc ción y crea ción ( praxis en el sen ti do de la póiesis). En sen ti do eti moló gi co, téc nica es tékne: "habi li dad", un saber-hacer - obtenido por experiencia y disposición corporal -. El saber-hacer está referido a la posesión de una habilidad simple o compleja, es decir, que va desde saber ejecutar una acción, hasta que dicha habilidad constituye una destreza.(1)

La actividad técnica, en cuanto un "hacer" se refiere a movimientos físicos concretos que conforman conjuntos coordinados de acciones. Pero en cuanto "saber" significa "distinción", "discernimiento", "diferenciación" y "elección” de lo que la sensibilidad confronta, lo cual confina al saber a un ámbito de inmediatez, de con ti güi dad con lo dado. Por lo ante rior, el ha cer de la téc ni ca nun ca es un mo vi mien to me cá nico, repetitivo y descualificado, sino que es un hacer que está siem pre, nece saria y esen cial men te de ter mina do porel saber. En el ser humano nunca es de otra forma.

Este carácter de inmediatez práctica y sensible que posee al saber-hacer, su capacidad de comprobar conocimientos pero no confundirse con ellos,(2) ha llevado a calificar como 'té- 
cnica', a todo instrumento, herramienta o procedimiento, en cuanto que éstos, para su interacción con los objetos están siempre subordinados por lo menos a un saber -a veces a conocimientos- que establece el cómo de su operación. La técnica-saber-hacer, saber-inmediato-está siempresubordinada a un complejo de fines humanos, sociales y comunitarios que son esencialmente mediatos y trascendentes dado su carácter comprensivo.

En este contex to, comprender a la clasificación bibliotecológica como una técnica, sólo es válido si la consideramos como un medio para los fines sociales que busca realizar la biblioteca.

Sin embargo, podrían señalarse otros elementos significativos para caracterizar a la clasificaciónbibliotecológicacomo una técnica, como sería su carácter utilitario y pragmático, que al gu nos es tu dio sos del tema han se ña la do. Pero esta consi de ra ción re quiere anali zar se, para ellocon vie ne ad ver tir sobre el uso ambiguo del término "clasificación". En algunos textos a dicho término se le utiliza con significado distinto den tro de un mis mo con tex to: así, “cla sifi ca ción" pue de re ferirse al sistema de clasificación que se está estudiando; a la clasificación lógica de los contenidos del sistema de clasifica ción; o se re fie re al acto de cla si fi car, como la prác ti ca en la que se lle va a cabo la cla si fi ca ción de los ma te ria les, se gún lo reglamenta el Sistema de Clasificación. En gramática la distinción entre 'clasificar' y 'clasificación' es obvia: un término de no ta al ver bo, y el otro al sus tan ti vo. En bi blio te co lo gía, dichos términos se refieren a actividades con distinto sentido.(3)

Correlativamente, por lo que a nuestro tema atañe, si queremos comprender si la clasificaciónes una actividad técnicao teórica, para su análisis tendremos que distinguir entre clasificar y sistema de clasificación.

Esta distinción, además de metódica pareciera ser histórica. Cla si fi car, se ha di cho, ha sido ta rea de los bi blio te ca rios desde la antigüedad, por medio del arreglo de los libros que respon de a la di vi sión e in te gra ción de los ma te ria les, de acuer do con la semejanza o diferencia de sus diversas características: alfabéticas, geográficas, lingüísticas, etc.Características que fueron diversificándose a medida que iba aumentando cualita tivay cuan ti ta ti va men te el ma te rialque po seía unabiblioteca.

Es a esta ac ti vi dad a la que ha cen re fe ren cia la ma yo ría de las denominadas definiciones de clasificacióncomo las siguientes: la de Margaret Mann quien dice que "en su comprensión más sencilla, la clasificación es la re u nión de co sas se me jantes; en su acepción más elaborada, es el arreglo de las cosas según sus semejanzas y diferencias".(4) Para W. S. Merril clasificar es el "arte de asignar a los libros un lugar determinado en un sistema de clasificación en el cual se agrupan, de acuerdo con sus semejanzas o relaciones, los diversos temas de la in ves ti ga ción hu ma na o las des crip cio nes de la vida hut mana en sus diversos aspectos".(5) John Dalton Dana dice: "clasificar libros es agruparlos incluyendo en cada grupo, en la medida en que sea posible, los que tratan de determinada materia, por ejemplo, de geología; o de todas las obras que versan sobre cual quierasun to pre sen ta doen forma particular, por ejem plo, la poe sía; o los que se re fie ren a de ter mi na do período de tiempo, por ejemplo, la Edad Media, etc. ..."(6) Por su parte, E. C. Richardson afirma: "la forma más completa y exacta de la definición de clasificación: la agrupación de cosas semejantes".(7)

Podemos advertir, que las definiciones anteriores describen acciones cuyos objetivos inmediatos son el acomodo de los libros u otros materiales en los estantes o anaqueles y su uso por los lectores. Es decir, no son definiciones.

De acuerdo con lo que señalamos sobre la actividad técnica, ve mos que las dis tin tas de fi ni ciones con sig na das des criben la actividad de clasificar con las características de la técnica, pues, la especifican como un conjunto de acciones prácticas relativas a objetos inmediatos -los libros-, en condiciones particulares y con cre tas -las bi blio te cas-. Em pero, son ac ciones que su po nen un sa ber de ri va do, no sólo de la ex pe rien cia inmediata con los diversos materiales, sino también de la elección entre las distintas formas de clasificar, de un tipo de arreglo adecuado a las necesidades in me dia tas de labiblio teca. Por lo mis mo, el su je to que cla si fi ca los ma te ria les de una biblioteca involucra en su habilidad un conjunto de posibilida des de ac ción, pues, sabercla sificares saberhacerypoder hacer una se rie de mo vi mien tos sig nifica ti vos y ade cua dos a: $1^{\circ}$ los materiales, $2^{\circ}$ a la forma de clasificación elegida para co lo car las obras en el lu gar que les co rres pon de, y $3^{\circ}$ a las necesidades de servicio de la biblioteca.

Com pren der la ac ti vi dad de cla si fi car como una ac ción téc nica, sólo es vá li do des de el en fo que an te rior, es de cir, que parte de definiciones laxas de clasificar, como las señaladas, y lue go ade cúa a ellas los da tos his tó ri cos so bre lo que ha cían y hacen los bibliotecarios cuando "acomodan" los libros. Sin em bar go, ese en fo que confunde la no ción de cla se con las nociones de "agregado" o de "todo", equiparando entidades concretasconentidadesabstractas. Las clases son entidades abas trac tas, aun cuan do los miem bros que las com po nen sean entidades concretas.(8)

Podría con si de rar se que el en fo que téc ni co de la cla si fi ca ción tie ne cier ta va li dezcuan do se re fie re a rea li da des es pe cíficas por lo que se denomina "clasificación utilitaria o práctica" (que en sentido estricto debiera siempre llamarse "forma de clasificar utilitaria"). Empero, es una validez condicionada por la necesidad, principalmente eco nó mi ca, en la que se encuentra la bi blio te ca y sólo en ca sos ex cep cio na les es ele gi da esta "clasificación utilitaria" fuera de este contexto; por ejemplo, cuando el carácter de la colección crea problemas peculiares para su almacenamiento, manipulación y préstamo, o cuan do se toma en cuen ta, de ma ne ra pre do mi nan te, el uso que dará a las obras el usuario.(9) 
La especificidad en que debe ser aplicado el enfoque técnico de la clasificación lo saca fuera del contexto de las reflexiones so bre los sis te mas de cla si fi ca ción, e in clu so de las que se re fie ren a la bi blio te ca, pues esas for mas de cla si fi car no po sibilitan alcanzar los fines de servicio comunitario de ésta ya que, como se ña la ra en 1930 la mis ma Mar ga ret Mann: los libros no son consultados porque ellos tengan un tamaño que co rres pon de a oc ta vos o fo lios, sino por que ellos tra tan de un asun to es pe cífico;(10) asun to que con for ma el fac tor cog nosci ti vo de ter mi nan te de la obra. Por lo tan to, de fen der un en foque técnico de la clasificación es, por un lado, asumir una con cep ción erró nea de lo que es un li bro (pura ex te rio ri dad) y lo que es una biblioteca (un depósito) con todas las implicaciones negativas que se derivarían sobre el carácter del bibliotecario, de finalidad y naturaleza. Por otro lado, vemos que este enfoque, que asu men va rios au to res so bre lo que denominan "el arte" de clasificar, y que autoriza determinar como téc ni ca a esta ac ti vidad, hace de lado su aspecto congnoscitivo que es el que permite denominarla con propiedad clasificación; ha cien do re fe ren cia a una ac ti vi dad teó rica que históricamente se ha desarrollado como tal.

Efectivamente, ha sido en el principio cognoscitivo, al que hace referencia el "asunto", "tema" o "contenido" de las obras, donde se han fundado las distintas clasificaciones que han existido desde las primeras bibliotecas. Esto se explica porque dicho "principio cognoscitivo" representa los diversos conocimientos, en sus múltiples aspectos, que ha produci dola ac ti vi dad hu mana. Las ca te go rías y con cep tos con que se han comprendido sus productos han conformado esquemasdelconocimiento humano, es de cir, ex presiones teóricas, abs trac tas; que se han en ri que ci do y trans for ma do conlain tegración y conceptualización de su estructura a lo largo de la historia del actuar humano.

Una primera in te gra ción y de no mi nación de los co noci mientos hu ma nos, se hace pre sen te en las bi blio te cas de la an ti güedad: pues, en la di vi sión y 'ac om odo' de las ta bli llas de la Biblioteca de Asurbanipal, lo que se está ordenando no son las tablillas en sí, sino el conocimiento que ellas contienen sobre el cielo, y las que se refieren al conocimiento sobre la tierra. En la Biblioteca de Alejandría los papiros se ordenaban de acuerdo al asun to que trataban: poe sía, ora toria, fi lo sofía, historia y miscelánea. En la Edad Media se da la misma actitud teó ri ca; en las bi blio te cas se di vi den las obras de acuer do con la significación ideológica de su contenido: por un lado las obras de he re jes, y por otro las de cre yen tes. Por el tipo de discurso que contienen: litúrgicas, eclesiásticas, de edificación, lec cionarios. Porelcarácter de susconocimien tos: his tóricas, geográficas, artes liberales, etc. En suma, no hay un resguardo de las obras por lo que po dría mos lla mar sus "cua li da des primarias" (color, for ma, ta ma ño), sino porsuscualificaciones abstractas, generales o universales.
Así, no solamente las clases en que se organiza la colección tienen carácter abstracto, que impide determinar a la actividad de clasificar como una técnica, sino que por lo anterior, tam po co la re la ción del su je to con el ob je to es prác ti ca, sen sible; pues to que el ob je to es tam bién abs trac to, pues, se cla sifica el contenido del libro (el objeto). Por lo tanto, la relación que se es ta ble ce en tre el su je to y el ob je to es una re la ción teó ri ca, men tal. Así como al cien tí fi co no se le pue de ca li fi car de técnico porque uti li ce lá piz y pa pel para ex pre sar sus ideas, o instrumentos de observación y verificación para comprobarlas; así tam po co al bi blio te ca rio se le pue de re du cir a téc ni co porque ten ga que colocar los obje tos físicos en los ana que les.

En la actividad de clasificaciónencontramos variosfactores, que deben volver precavidos a quienes pretendan continuar mermando esta actividad al confundirla con las actividades técnicas, que son:

a) Las cla ses en que se agru pan las obras tie nen por na tu ra le za un carácter abstracto.

b) El objeto que se clasifica, el contenido de la obra, es abstracto.

c) La relación que tiene el sujeto con el objeto es teórico. No se puede tener una relación práctica-sensible con abstracciones.

Por lo anterior, podemos afirmar que la clasificación es una actividadteóricaque es tablecelamediación delpen sarentre elsuje toconcreto in dividualyelobjetofísico, cual quie ra que éste sea (obras, colección,materiales, documentos, soportes, etc.).

\section{DE LO TEORICO}

Resultanormalmente cómodo calificar a los sistemas de clasificación elaborados en el siglo XIX como sistemas lógicos del conocimiento; y a los anteriores, como clasificaciones prácticas y uti li tarias. Este en fo que, por lo an te rior men te expuesto, no resulta verificable, sobre todo por los connotados ejemplos que lo contradicen.

Laclasificaciónque rea li zaCalíma co en la Bibliote cade Alejan dría con sus cin co di vi sio nes por ma te ria era una cla si ficación ló gi ca cuyo es que ma fue de ri va do de la ló gi ca pla tónica yaristotélica; asimis mo, la BibliotecaUniversalis de Kon rad Von Ges ner pu bli ca do en 1545- 1555 es un ca tá lo go de obras en griego, latín y hebreo clasificadas de acuerdo con principios lógicos definidos. 
Sin em bar go, hay un ele men to fun da men tal que sí dis tin gue a las clasificaciones exis ten tes y que se manifies taconclaridad dentro de lo que habría que denominar: sistemas simbólicos de clasificación. Este elemento es el origen desde donde se generan dichos sistemas, pues, unos tienen como base de organización de los co no ci mien tos es que mas ex ter nos alámbito bibliotecario, y otros parten de la necesidad de organización de la colección dentro de cada biblioteca. Probablemente, el que inicia esta transición es W. T. Harris, quien concibe, en 1870, un esquema de clasificación para la biblioteca de la escuela pública de St. Louis, invirtiendopara ello, se ha dicho, el esquema de clasificación de Bacon para adaptarlo mejor a las necesidades de su colección.

Se podría afirmar que ese origen de la clasificación bibliotecológica, al estar determinado por una necesidad particular - organizar una colección específica-, cualifica a la clasificación como una actividad práctica y no teórica, técnica y no científica; in de pen diente men te de la na turale za teóricadelos ele men tos con los cua les se ela bo re una res pues ta a di cha necesidad y de qué modo se utilicen sus resultados. Así por ejem plo; el con di cio na mien to por la ne ce si dad lle va a Ha rris a no utilizar estrictamente el sistema filosófico de clasificación de las ciencias de F. Bacon sino solamente aquello que considera más con ve nien te y ade cua do para ela bo rar sus sistema, pues sólo toma en cuenta las actividades básicas que fundamentan el sistema de Bacon -historia filosófica y poesía-, ha cien do evi den te lo que dice Sa yers: "los sis te mas fi losóficos de clasificación de las ciencias han tenido alguna influencia en la clasificación de bibliotecas pero la conveniencia práctica fue siempre un factor muy significativo". ${ }^{10}$

Podríamos argumentar, sin embargo, que si bien toda actividad téc ni ca tie ne su ori gen en una ne ce si dad con cre ta, no toda necesidad práctica se ha de satisfacer a través de actividades técnicas. En esta situación están Harris, Dewey, Blis, etc., quienes enfrentando un problema práctico, técnico, lo resolvieron, sin embargo, teóricamentecon Sistemas de clasificación, cuya utilidad general está manifiesta en su estructura cognoscitiva, de principios, leyes y reglas generales para la explicación, regulación y uso de sus objetos. Por ello, el que la mayoría de los sistemas de clasificación bibliotecológicos se originen en el contexto de una biblioteca específica, no es obstáculo para que sean aplicados en cualquier biblioteca dada o posible.

Por lo an te rior, re sul taim por tan te en tre sa carotras carac te rísticas teórico-científicas de la clasificación como actividad humana:

Estas características pertenecen al ámbito de la lógica y permiten afirmar que los sistemas de clasificación tienen como propiedad esencial su naturaleza lógica que los dota de una cualidad eminentemente teórica:
En primer lugar, la clasificación es un pensamientocontrolado y, por lo mismo, un actuar notablemente metódico que produce la organización de hechos aprehendidos originariamente de manera discordante e inconexa, como serían los libros en sus diferentes tamaños y contenidos, o la diversidad de ma te ria les que ad quie re una biblio te ca. Los dis tin tos objetos que componen dichos materiales, particularmente los libros, se ha cen aco plar unos con otros en una co lo ca ción or denada que produce lo que, con relación al punto de partida, puede considerarse un sistema.

El or den no es lo mis mo que el sis te ma. Lo que está or de na do son los libros particulares. El sistema es la colocación ordenada resultante. Por ello, una dis po sicióno cla se de li bros no es en cuan to tal, una dis po si ción or de na da. Es de cir, el or den no se logra mediante una disposición ordenada. Es decir, el orden no se logra mediante la disposición en clases, puesto que, si todo lo que sa be mos de un li bro es que per te ne ce a una clasecuya propie dad de fini doraconocemos, en tonces, loque sabemos de ese libro está limitado a lo que puede deducirse de esa pro pie dad de fi ni do ra. El sa ber acer ca de otros li bros no nos da un saber adicional de ese libro. Con una disposición ordenada sucede de otra forma.

LaCla si fi ca ción De ci mal de Dewey es un sis te ma por que posee una disposición ordenada con carácter deductivo, es decir, es je rár qui ca. Ade más, el con jun to de li bros de una co lección clasificada bajo el sistema de Dewey exhibe orden porque, da das las pro pie da des de al gu nos miem bros del conjunto, los otros miembros quedan determinados. Esta determinación se debe a la relación jerárquicay de ci mal que or dena al conjunto, la cual no es propiedad de los libros considerados como una clase, puesto que los libros pueden ser de dis tin tas cla ses, pero el or den en que es tas cla ses se organizan es lo que constituye el sistema. De tal manera, que del orden en que se divide el conocimiento, por disciplinas ge ne rales, po de mos in fe rir el lu gar que co rres pon de a las disciplinas particulares. El sistema permite continuar las subdivisiones de la disciplina hasta el grado deseado. En Dewey, "las diez pri me ras cla ses que sir ven de base al sis te ma se subdividen cada una en nuevos núcleos de clasificación de segundo grado, cada una de las divisiones de segundo grado pue de sub di vi dir se a su vez de ci mal men te, dan do ori gen a las clasificaciones de tercer grado. Y así sucesivamente".(11)

Resulta por demás repetitivo señalar las características del sis te ma de Cut ter, Blis, L.C., Ra ga nathan, etc., en cuan to que todas ellas exhiben una disposición ordenada de las disciplinas que las in te gran, aun que exis ten di fe ren cias en cuan to a la estructuradel sis te maque em plean. Señale mos someramente dichas diferencias:

Cutter emplea tablas de clasificación constituidas por clases básicas que incorporan numerosas subclases y subdivisiones e incluyen el desarrollo alfabético de ciertos temas. Este sistema se especifica, además, por su carácter expansivo en cuanto que sus tablas de clasificación preveen el desarrollo 
cuan ti ta ti vo de las obras de una bi blio te ca y su pro bable rit mo de crecimiento.

Por su par te, el sis te ma de cla si fi ca ción de Hen ry Evalyn Blis se caracteriza porque, el orden de las distintas clases del conocimiento establece una relación vertical de subordinación, y una coordinación horizontal entre ellas; además de que sus "tablas auxiliares" son comunes para todo el sistema, y en otras oca siones, "es pe cíficas" para una ma te ria, como su ce de con la subdivisión numérica "que puede anteponerse o posponerse a cualquier cla se dan do lu gar, en un caso, a la de partamentalizacióndecolecciones y en el segundo, a la subdivisión de forma”.(12)

Bástenos estos ejemplos para señalar que el orden es la relación que guardan los miembros de una clase, que hay diversos ti pos de or den y di ver sos gra dos en que se pue de hallar el ordenamiento. Es necesario también remarcar, con respecto de los distintos sistemas de clasificación bibliotecológicos, que la introducción del orden de lo que está ordenado puede tener todos los grados de complicación posibles, y dichas complicaciones pueden ser de diferentes clases, pero todas ellas suponen la acción del pensamiento reflexivo, de la teoría, del alejamiento metódico de las apariencias.

La disposición ordenada como factor determinante del sistematie ne en las cla sifi ca cio nes biblio te coló gi cas, la mis marazón de ser que en el pensamiento científico en general: la comprensión de los constituyentes de una situación fáctica, que en nuestro caso son los materiales de las bibliotecas.

El pri mer paso en la com pren sión se lo gra a tra vés de la ge nera li za ción, es de cir, me dian te la abs trac ción que está im pli cada en la co ne xión de un he cho con otro que se le ase me je. Para proce der a la ge ne ra li za ción es ne ce sa rio que el co no ci miento, constituyente básico de la situación fáctica, sea agrupado en cla ses para ser or de na das en tre sí y ob te ner de esta ma ne ra una generalización creciente.(13)

La ge ne ra li zación rea li za da se evi den cia en las dis tin tas notaciones en que se expresan simbólicamente los sistemas de clasificación bibliotecológicos; generalización que implica dos ni veles: en pri mer lu gar, la no ta ción mis ma, sea nu mérica o alfabética, es un sistema abstracto de representaciónde las di vi sio nes y sub di vi sio nes en las que se ha ana li za do el co nocimiento. En segundo lugar, en el momento en que las obras se clasifican por materias, es decir, por su contenido cognosci ti vo y no por sus ca rac te rís ti cas fí si cas, se ha rea li za dootra abstracción: la selección de algo que también está presente para ser observado; puesto que, el reconocimiento de la propie dad cog nos ci ti va que po see el li bro en traña el ser aprehendido en abstracción de otras propiedades con las que, de hecho, ésta es dada, como la propiedad material, formal, tonal, etc.

En ter cer lu gar, el uso de "nom bres de cla se de fi ni dos" in di ca también que se ha hecho una abstracción más, por medio de una se rie de ope ra cio nes ló gi cas pre su pues tas en toda ac ti vidad de clasificación los libros son un con jun to de ob je tos que han sido agrupados, porque cada uno de ellos posee ciertas propiedades, las cuales son las importantes y no la cosa sustantiva, libro, del cual se dice que son propiedades. La agrupación de los li bros se hace, por tan to, por la se me jan za en tre las propiedades que los definen, agrupación que va a conformar las distintas clases. Los nombres de cada clase expresan las especies de propiedades agrupadas. Dos o más especies pue den te nerca rac te rís ti cas en co mún que hacen po si ble consi de rar que las dos es pe cies jun tas cons ti tu yen una cla se. Tal clase es un género. Una especie de un género es disímil de otra es pecie por una dis tin ción carac te rís tica. Esta carac terística es lo que Aristóteles significó por diferentia (Diaforá).(14) Una di fe ren cia es la pro pie dad de fi ni do ra de una cla se. La definición de una especie por género y diferencia muestra a la especie en relación con otras especies coordinadas con ella en el mismo gé ne ro. Pero, si el gé ne ro pue de ser definido, en tonces, es una es peciere la ti va a al gún otrogéne ro y así sucesivamente. Por ejemplo: la clasificación de la Biblioteca del Con gre so, con sus cla ses: Obras Ge ne ra les, Fi losofía, Religión, Historia, etc., y sus géneros: Ciencias sociales: general, estadística y economía; teoría económica, etc. Semejante organización ordenada bajo géneros más y más amplios constituye una clasificación o sistema clasificador, como lo son todas las clasificaciones bibliotecológicas.

\section{DE LO SIMBOLICO}

Los Sistemas de Clasificación Bibliotecológicos, si bien tienen como objetivo concreto: el ordenamiento físico de los materiales de una biblioteca, esencialmente son sistemas lógico-simbólico de ordenamien to de diversos materiales. Este carácter lógico-simbólico de los sistemas de clasificación es otro de los factores de cientificidad de la bibliotecología.

La ciencia, en sentido estricto, nace de la fundamentación pla tó ni ca de la ló gi ca, la cual se con si de ra como una es fe ra de la razón humana que "investiga los requisitos esenciales del saber 'auténtico' y de la ciencia 'auténtica', concebida por ende como una exposición de las nor mas con for me a las cuales pue da cons truir se una cien cia que as pi re cons cien te men te a le gi ti mi dad nor ma ti va ge ne ral y que con secuen te men te justifique su método y su teoría”.(15)

En este sentido la lógica, en cuanto proceso de exhibición de las nor mas para cons truir un co no ci mien to uni ver sal, se consti tu ye en el an te ce den te a los mé to dos prac ti ca dos por la ciencia y a las cien cias exis ten tes, sir vien do de guía práctica para dichos métodos y ciencias. El sentido de la lógica, por tanto, "no con sis te en to mar por nor ma elfactum de al gún mé to do o el de alguna ciencia desarrollada con ingenuidad, para dar forma legítima a las operaciones científicas".(16) La lógica sería para Platón, en términos modernos, una teoría de la ciencia. 
Por ello la ló gi ca, que tuvo su ori gen en el pen sa mien to pla tónico, forja con la analítica aristotélica una teoría sistemática rígidamente conformada que persiste a través de los siglos.

Pero en la época moderna, época en que las ciencias se indepen dizan para conver tir se en cien cias es pe ciales, la ló gi ca,lejos de procurar una guía fundamental a las ciencias se deja guiar en su ideal científico y en el planteamiento de sus problemas por las ciencias fácticas, especialmente por las ciencias naturales. Aunado a ello, vamos encontrando en el silogismo, forma sustantiva de los procedimientos lógicos, una incorporación en la matemática formal, que se conjunta con la ela bo ra ción de una ál ge bra si lo gís ti ca, que na ció de los inte re ses de la téc ni ca teó ri ca de duc ti va de la cien cia ma te mática, en la matemática inglesa de principios del siglo XIX (De Morgan, Boole); lo que implicó una transformación en la silogística, convirtiéndose en una "lógica extensiva" que, haciendo a un lado los artificios de toda clase para hacerla inofensiva para la práctica del teorizar matemático, contiene un núcleo de pensamiento que posee su propia legitimidad: el análisis formal.(17)

La generalización de la lógica ha dado como resultado una ciencia de la forma pura que no puede ya distinguirse de las matemáticas: la lógica matemática. Así vemos, como dice Ramsey,(18) que la palabra "lógica" en la actualidad se maneja con dos significados: la lógica como ciencia del orden, susceptible de expresión en un sistema simbólico, y la ló gica como análisis y crítica del pensamiento.

Los sis te mas de clasifi ca ción biblio te coló gi cos, da das sus características fundamentales, pertenecen a los sistemas lógicos- simbóli cos; aun que al gu nos man ten gan, en cier to gra do, elementos de la lógica tradicional como serían aquellos que tienen un sistema de clasificación interno por género y especie, como en el caso de Dewey, lo cual resulta comprensible por la época en que fue elaborado. Sin embargo, el sistema po see una no ta ción pura de nú me ros que le asig nan un ca rácter formal, es decir, matemático; por ello podemos afirmar que, en cierto sentido, las clasificaciones de Cutter, Bliss, Ranganathan, DU, y L.C., tienen su fundamentación última, en el proyecto Leibniziano de un cálculo del razonamiento (19) que concluyedirectamente en la con cep ción de un sis tema simbólico cuya significación está determinada por reglas de com bi na ción y es así, in de pen dien te de cual quier in ter preta ción del sis te ma. El sis te ma sim bó li co es lo que se lla ma comúnmente lógica simbólica, y sus fundamentos fueron establecidos por George Boole, como señalamos anteriormente. Boole afir mó en 1847 que "aque llos que es tán fa mi lia ri za dos con el estado actual de la teoría del álgebra simbólica están conscientes de que la validez de los procesos del análisis no dependen de la interpretación de los símbolos que se emplean, sino úni ca men te de las le yes de su com bi na ción. Todo sistema de interpretación que no afecte la verdad de las relaciones supuestas es igualmente admisible, y es así como el mis mo pro ce so pue de, bajo un es que ma de in ter pre ta ción representar la solución de un problema sobre las propiedades del nú me ro; bajo otro es que ma, pue de re pre sen tar la so lu ción de un problema geométrico; y bajo un terceresque ma, re presentar la solución de un problema de óptica”.(20)

Quie nes a su vez es tán fa mi lia ri za dos con los sis te mas de clasificaciónbibliote cológicos, endondelos diferentes sistemas de no ta ción, mix tos o pu ros, son in de pen dien tes de los pro cesos de análisis del conocimiento que utilizan, comprenderán con mayor claridad las palabras de Boole, sobre todo si establecemos las diferencias de dichos sistemas en las leyes de combinación de los símbolos que implican. Así, las leyes de combinación del sistema de Dewey, esencialmente jerárquico en su notación, como también entre las disciplinas y los asun tos, es table ce la di vi sión su ce si va de la dis ci pli na o asunto y la re pre sen ta por la adi ción de otro nú me ro dí gi to a la nota ción sig ni fi ca ti va. Esto se mues tra con cla ri dad en el es quema, haciendo que el encabezamiento de cada nueva subdivisión explicite su subordinación a un concepto más am plio por me dio de la san gría, la cual va ria rá se gún la ex tensión del número. Otra ley de combinación de símbolos en Dewey se puedeejemplificar cuan do se se ña la que todo concepto expresado por la notación más específica que la de la clase principal está subordinada a todos los conceptos más am plios de los cua les es una par te. Por ejem plo, lo que es cierto y se aplica a la clase principal 600 , es también cierto para todas sus subdivisiones, o lo que se aplica a la clase 631, es cier to para to das sus sub di vi sio nes, y asísu ce si va men te has ta la más minuciosa de las subdivisiones.(21)

Las leyes de combinación de símbolos están expresas también en las dis tin tas re glas para usar la no ta ción; por ejem plo: la que señala cómo usar más de un cero en las subdivisiones comunes; cómo añadir o expandir ciertos números para hacer los más es pe cífi cos; cuál es el va lor je rár qui co de las no tas de adi ción, etc..(22) Re glas que es tán im plí ci tas en los su marios, los esquemas, el índice y las tablas auxiliares.

Por su parte, el Sistema de Clasificación Decimal Universal combina sus símbolos con reglas más específicas por cuanto que és tas re pre sen tan: tan to el tema que tra tan las obras a clasificar, como sus características especiales, combinando las tablas principales con otras auxiliares no usadas en su totalidad por la CD (clasificación de Dewey). Su introducción en el símbolo clasificador exige, además, signos adicionales tales como paréntesis, comillas, etc.(23)

Particularmente, el Sis te ma de Cla si fica ción de la Biblio te ca del Con gre so en Wa shing ton es qui zá una de las ex pre siones más acabadas de la utilización de la lógica simbólica en la clasificación bibliotecológica.

Con un sis te ma sim bó li co de no ta ción mix to, por cuan to que utilizanúmeros y le tras e im pli ca una com bi na to ria de las características de los dos sistemas decimales: el de Cutter y los especialistas que cooperaron con él, y la Clasificación Francesa de Brunet. "El sistema agrupa las materias más generales por medio de 21 le tras (omi te i, o, w, x, y, que se re servan 
para futuras ampliaciones) y luego las subdivide combinándolas con otras 21 letras para formar las materias principales ... Cada ma te ria prin ci pal se sub di vi de por me dio de nú me ros cardinales (no decimales) según un plan general que, de acuerdo con la riqueza bibliográfica de la materia, se emplea parcial o totalmente".(24)

Este sistema simbólico tiene como característica general ser en símis mo un con jun to de sub sis te mas simbóli cos, en don de cada uno establece sus propias leyes de combinación. El no haber comprendido esta característica, propia de la teoría de con jun tos de la ló gi ca sim bó li ca, ha lle va do a afir mar que "la clasificación de la Biblioteca del Congreso (LC) no es realidad un sis te ma de cla si fi ca ción sino un con jun to de cla si fi caciones".(25)

En la misma situación que L.C. se encuentra el "Colon Classification" de S.R. Ranganathan. Este es también un sistema simbólico con mayor precisión matemática, por cuanto que sus subsistemas simbólicos son realmente microsistemas unitarios con sus propias variables simbólicas (números, letras mayúsculas y minúsculas del alfabeto romano, letras griegas, signos de puntuación). "Estos signos dan origen al lenguaje artificial del sistema (Colon Language), donde el cero, los signos de puntuación y las flechas desempeñan el papel de conjunciones, mientras que los demás signos actúan como sustantivos".

Baste lo anterior para señalar, finalmente, que la estructura teórica de los sistemas de clasificación analizados, su carácter lógico simbólico, determinan a la actividad de clasificación comoun trabajora di cal men te teó ri co que a di fe ren cia de las teorías sobre la clasificación cuya finalidad es solamente comprensiva y explicativa; los sistemas de clasificación bibliotecológicostienen como meta precisa el ser usados prácticamente. Son la respuesta a problemas concretos, específicos y particulares a través de estructuras teóricas que en virtud de su generalidad pueden ser aplicados en bibliotecas de distinta índole y de diversos lugares.

\section{CONCLUSIONES}

La reflexión filosófica que hemos intentado realizar sobre el pro ble ma del ca rác ter teó ri co o téc ni co de la ac ti vi dad de clasi fi ca ción en las bi blio te cas se ha de sa rro lla do en los si guientes niveles:

1. El que corresponde a la actividad misma de clasificar las colec ciones de unabiblio te ca con un sis te ma de cla sifica ción.

2. El que se refiere a la actividad de elaborar un sistema de clasificación bibliotecológico.

3. El relacionado con el origen de los Sistemas de Clasificación y,

4. El vin cu la do a las me tas que per si guen los Sis te mas de Cla sificación.

El análisis de estos niveles nos ha mostrado el carácter específicamente teórico deestaactividad, independientementede las cir cuns tan cias que la ori gi nan y las me tas a las que se di rige.

Sin embargo, existe un espacio dentro del cual la actividad teórica de clasificación es desvirtuada y desnaturalizada: el ámbito de su aprendizaje mnemotécnico; al que suele reducírsele en circunstanciasescolares o bajo actitudes pragmáticas y efi cien tis tas. En este con tex to, re sul ta su perfluo de mostrar filosófica o científicamente la estructura teórica de una ac ti vi dad, sus ni ve les de profun di dad abs tracta, ge ne ral y univer sal, o el va lor mis mo de la di men sión teó ri ca como vi sión más amplia y comprensiva del mundo y sus fenómenos, si el sujeto que la realiza, o el que obliga o in du ce a apren der la, la reduce a un contenido puramente repetitivo o simplemente descriptivo.

En suma, po de mos con cluir afir man do que: lo teó ri co o téc nico de la actividad de clasificación bibliotecológica, no sólo radica en su estructura interna sino, fundamentalmente, en la "estructura formativa" de quien la realiza o la transmite.

\section{NOTAS}

1.- $\quad$ VILLORO Luis, Creer, Saber y Conocer, SXXI, México, 1981, p. 127

2.- $\quad$ Ibídem, p. 128

3.- KRISHAN KUMAR, Theory of Classification, Third Edition, VIKAS PUBLISHING HOUSE PVT LIT, Naveen Shahdara, Delhi, pp. 2-3. (Ranganathan, por ejemplo, distinguió cinco sentidos en que se emplea el término "Clasificación”).

4.- MARGARET MANN, Introduction to Catalogingand the Classification of Books, American Library Association, Chicago, 1930, p. 41 
5.- $\quad$ Ci ta do por: PEN NA CAR LOS V, Catalogación y Clasificación de Libros, 2da. ed. corregida y ampliada, Kapeluz, Buenos Aires, 1964, p. 139

6.- $\quad$ Citado por: LITTON GORDON, Clasificación y Catálogos, Bauker, Buenos Aires, 1971, p. 8

7.- $\quad$ RICHARDSON, Classification, Scribner, New York, 1912, p. 1

8.- $\quad$ FERRATER MORA, Diccionario de Filosofía, F.C.E., México, 1969. Artículo sobre Clasificación.

9.- CUSTER A. B, Introducción, (Comentarios sobre la filosofía, la estructura y el uso de la clasif. de Dewey. Editor, 18a. XXIV

10.- MARGARET MANN, op.cit.p. 40

11.- PENNA CARLOS V., op.cit., pp. 154-155

12.- $\quad$ Ibídem, p. 180

13.- Cfr. STEBBING.L.S, Introducción Moderna a la Lógica, CENTRO DE ESTUDIOS FILOSOFICOS, UNAM, 1965 , p. 269

14.- $\quad$ ARISTOTELES, Metafísica, 122b

15.- HUSSERL EDMUND, Lógica Formal y Lógica Trascendental. FCE p. 5

16.- Ibídem.

17.- $\quad$ Ibídem, pp. 75-75

18.- RAMSEY, F. P. Proc., London Matematical Association, series z vol. 25, p. 353 a.

19.- LEIBNIZ G. F., Nuevo ensayo sobre el entendimiento humano, AGUILAR México, 1971, Libro I, cap. II, p. 22

20.- $\quad$ BOOLE G. The Mathematical Analysis of Logic. MacMillan, 1947, p. 3

21.- CUSTER A. B., op.cit., p. XXXIII

22.- Ibídem, p. xli, xlii y xliv

23.- $\quad$ LITTON GORDON, op. cit., p. 153

24.- PENNA CARLOS V. op. cit., pp. 174-175

25.- $\quad$ Ibídem, p. 174 